\title{
The Effect of Surfactants on the Diameter and Morphology of Electrospun Ultrafine Nanofiber
}

\author{
Jian-Yi Zheng, ${ }^{1}$ Ming-Feng Zhuang, ${ }^{1}$ Zhao-Jie Yu, ${ }^{1}$ Gao-Feng Zheng, ${ }^{1}$ Yang Zhao, \\ Han Wang, ${ }^{2}$ and Dao-Heng Sun ${ }^{1}$ \\ ${ }^{1}$ Department of Mechanical and Electrical Engineering, Xiamen University, Xiamen 361005, China \\ ${ }^{2}$ Guangdong Provincial Key Laboratory of Micro/Nano Manufacturing Technology and Equipment, \\ Guangdong University of Technology, Guangzhou 510006, China
}

Correspondence should be addressed to Yang Zhao; zhaoy@xmu.edu.cn

Received 27 February 2014; Revised 29 May 2014; Accepted 29 May 2014; Published 24 June 2014

Academic Editor: Ruigang Liu

Copyright (C) 2014 Jian-Yi Zheng et al. This is an open access article distributed under the Creative Commons Attribution License, which permits unrestricted use, distribution, and reproduction in any medium, provided the original work is properly cited.

\begin{abstract}
Different surfactants are introduced to study the diameter and morphology transformation characteristics of electrospun nanofiber. Surfactants increase the net charge density and instability motion of charged jet. The instability motion provides a good way to stretch the charged jets into finer ones, by which the beaded structures are also prevented. Ultrafine nanofiber with average diameter less than $65 \mathrm{~nm}$ can be fabricated. The nanofiber diameter decreases with the increase of surfactant concentration in polymer solution. The nanofibers with anionic surfactant sodium dodecyl sulfate (SDS) have the smallest diameter. The cationic surfactant hexadecyl trimethyl ammonium bromide (HTAB) plays the best role to prevent the formation of beaded structures in nanofibers, and helps to increase the uniformity of electrospun nanofiber. The effects of surfactants on the nanofiber diameter and morphology have been studied, which would promote the industrial application of ultrafine polymeric nanofibers.
\end{abstract}

\section{Introduction}

Ultrafine polymeric nanofibers have wide application in the fields of micro/nanosystem and flexible electronics. At present, quick and low cost fabrication are the main aspects for the development and industrial application of ultrafine polymeric nanofibers, which have attracted a lot of attentions in recent years $[1,2]$. As a unique and simple nano/micromanufacturing technology, electrospinning does not require stencil-plate, photo etching, or ultra clean chamber. Nanofiber with diameter ranges from several nanometers to several micrometers [3] can be electrospun at normal pressure and temperature. Electrospinning also has good materials compatibility that meets the development demands of polymeric, flexible, and bio-nano/microsystem. By now, hundreds of materials have been electrospun into nanofibers through electrospinning [4, 5], such as polymer, glass, metal, and protein. Electrospun nanofiber has extremely high surface area and outstanding mechanical and physical properties, which are suitable for a wide range application fields, such as drug delivery [6, 7], sensing materials $[8,9]$, catalyze template [10], and ion battery membrane [11, 12]. Decreasing the diameter and promoting the uniformity of electrospun nanofiber are the key roles for their industrial application.

During the electrospinning process, the high voltage is applied to stretch viscoelastic solution into the cone shape named as "Taylor Cone." When the electrical field force overcomes the surface tension, a jet is ejected from the cone tip. The charge repulsion force that stems from the accumulated charges imports disturbances into the ejection and motion process of polymer jet. The instability motion is the important factor to stretch and thin the charged jet [13]. Attributed to the bending and whipping instability, electrospinning nanofiber deposited randomly on collector as nonwoven.

The morphology of electrospun nanofiber is an important factor to investigate the rheology behaviors of charged jet in the ejection process. With the inadequate stretching of liquid jet, there are usual beaded structures appearing along 
the electrospun nanofiber. The beaded structures stem from the competition surface tension of liquid jet [14], in which the solution viscosity, surface tension, and net charges are the main influencing factors [15]. Repulsion force among electrospinning jets increases with the increase of charge density and provided an effective way to increase the uniformity and decrease the diameter of electrospun nanofiber. The surfactants have been introduced to decrease the surface tension and increase the net charge density. As such, Lin et al. [16] used cationic surfactants to increase the jet charge density; then instability motion can be enhanced and the beaded nanofibers were also overcome; Jia and Qin [17] used surfactants to change the surface tension of electrospinning solution; then the thermal performance and inner structure of nanofibers can be adjusted. The influences of surfactants on the diameter and morphology should be studied further to promote its application.

In this work, different surfactants were introduced to decrease the nanofiber diameter and prevent the formation of beaded structures in electrospun nanofiber. The surfactants would increase the net charge density and enlarge the repulsion force among charged jets. Then, the instability motion was also enhanced by the charge repulsion fore. The transformation characteristics of nanofiber diameter and morphology were studied.

\section{Experiments Details}

2.1. Experiment Setup. The electrospinning setup based on conventional pole-type nozzle configuration was built up in this work. The high voltage source (DW-P403-1AC, Tianjing Dongwen High Voltage Power Supply Plant, China) was used to provide electrical field between steel nozzle spinneret (inner diameter was $232 \mu \mathrm{m}$ and outer diameter was $500 \mu \mathrm{m}$, Shanghai Kindly Enterprise Development Group, China) and aluminum paper collector (thickness $0.04 \mathrm{~mm}$, Zhongshan Sinon Daily Products Co., Ltd., China). The anode of the high voltage source was connected to the steel nozzle spinneret, and the cathode was connected to the grounded collector. The precision syringe pump (Harvard 11 Pico Plus, USA) was used to transfer polymer solution from syringe to spinneret.

2.2. Electrospinning Solution. Polyvinylidene fluoride (PVDF, average molecular weight $=141,000 \mathrm{~g} / \mathrm{mol}$, DuPont, USA) solution was used as electrospinning solution. PVDF powder was added to the mixed blends of acetone (Sinopharm Chemical Reagent Co. Ltd., China) and N, N-dimethylformamide (DMF, Sinopharm Chemical Reagent Co. Ltd., China). The weight ratio of acetone to DMF in the blending solvent was $2: 3$. The PVDF concentration in the solution was $12 \mathrm{wt} \%$.

Anionic surfactant sodium dodecyl sulfate (SDS), nonionic surfactants Triton X-100, and cationic surfactants hexadecyl trimethyl ammonium bromide (HTAB) were added to the PVDF solution to investigate the effect of surfactants on the diameter and morphology of electrospun nanofiber, respectively. All of these three surfactants were purchased from Sinopharm Chemical Reagent Co., Ltd. China, which were used without any further purification. The surfactant concentrations in the PVDF solution were $3.5 \times 10^{-3} \mathrm{~mol} / \mathrm{L}$, $1.75 \times 10^{-2} \mathrm{~mol} / \mathrm{L}$, and $3.5 \times 10^{-2} \mathrm{~mol} / \mathrm{L}$, respectively.

2.3. Electrospinning Process. In the experiment, the PVDF solution was transferred to the nozzle spinneret by the precision syringe pump at a flow rate of $200 \mu \mathrm{L} / \mathrm{hr}$. High voltage of $13 \mathrm{kV}$ was applied at the nozzle spinneret, and the distance between spinneret to collector was $15 \mathrm{~cm}$. The electrospun nanofibers were observed and measured by a SEM (LEO 1530 field emission scanning electron microscope). Before the observation of SEM, the samples were sputter-coated with a gold layer of about $10 \mathrm{~nm}$ thickness.

\section{Results and Discussion}

3.1. Solution Characteristics. Firstly, the characteristics of polymer solution with surfactants were tested. The viscosity of PVDF solution was $153 \mathrm{mPa} \cdot \mathrm{s}$, which was the same as the polymer solution without surfactant. Surfactants can increase the free charges in polymer solution and conductivity of solution. The conductivity of polymer solution increased from $138 \mu \mathrm{s} / \mathrm{cm}$ to $860 \mu \mathrm{s} / \mathrm{cm}$, as SDS surfactant concentration increased from $3.5 \times 10^{-3} \mathrm{~mol} / \mathrm{L}$ to $3.5 \times 10^{-2} \mathrm{~mol} / \mathrm{L}$. And the conductivity of polymer solution increased from $167 \mu \mathrm{s} / \mathrm{cm}$ to $1104 \mu \mathrm{s} / \mathrm{cm}$, as HTAB surfactant concentration increased from $3.5 \times 10^{-3} \mathrm{~mol} / \mathrm{L}$ to $3.5 \times 10^{-2} \mathrm{~mol} / \mathrm{L}$. The relationship between conductivity of polymer solution and surfactant concentration was shown in Figure 1. But the Triton X-100 did not change the conductivity, since the Triton X-100 was nonionic surfactant and did not increase the free charges in polymer solution. Figure 1 showed that the conductivity did not increase with the surfactant concentration. The conductivity of PVDF solution with Triton X-100 was $9 \mu \mathrm{s} / \mathrm{cm}$, which was the same as the polymer solution without surfactant.

3.2. Beaded Nanofiber without Surfactants. Then, PVDF solution without surfactants was used as electrospinning solution. Due to the fast evaporation of solvent, the charged jets cannot be stretched adequately into fine and uniform ones. Liquid jet would be shrank into beaded structure by the surface tension, as shown in Figure 2 . The beaded nanofibers with a diameter range from $30 \mathrm{~nm}$ to $170 \mathrm{~nm}$, and the average diameter was $67.02 \mathrm{~nm}$. The standard deviation of electrospinning nanofiber was $31.62 \mathrm{~nm}$. Attributed to the large surface tension and small stretching ratio, the electrospinning nanofiber had large diameter distribution. The beaded structure on the electrospinning nanofibrous web also had large diameter distribution, which ranged from $422 \mathrm{~nm}$ to $6.28 \mu \mathrm{m}$. And the average diameter of beaded structure was $2.06 \mu \mathrm{m}$. The diameter distribution range of nanofiber electrospun from PVDF solution without surfactant was shown in Figure 3. Decreasing the surface tension and solution viscosity provided a good way to overcome the beaded structure. On the other hand, increasing the net charge density and electrical field forced applied on the charged jet would also promote the stretching and uniformity of charged jet. 


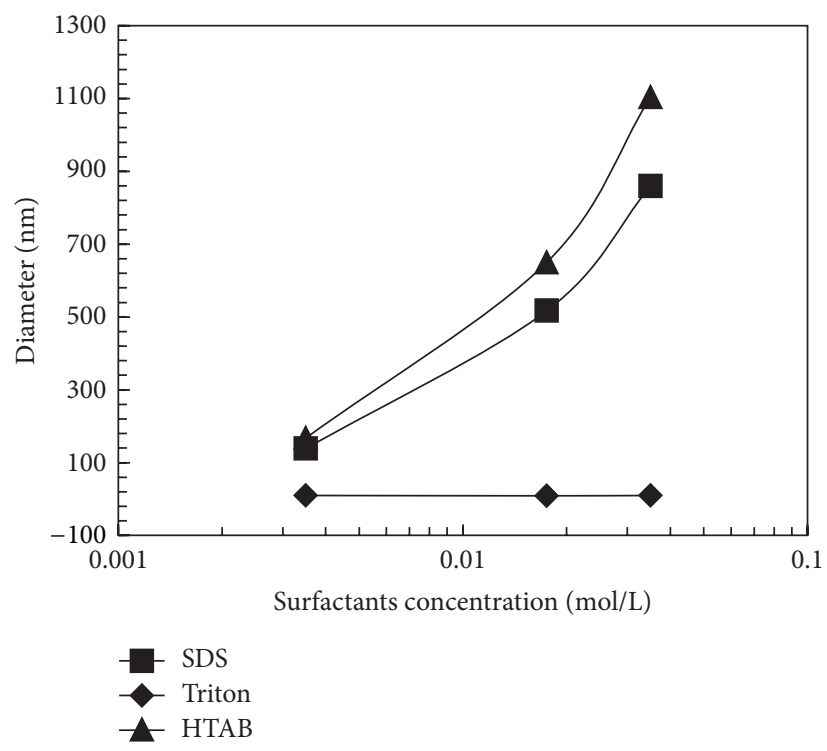

FIGURE 1: The relationship between solution conductivity and surfactant concentration in the solution.

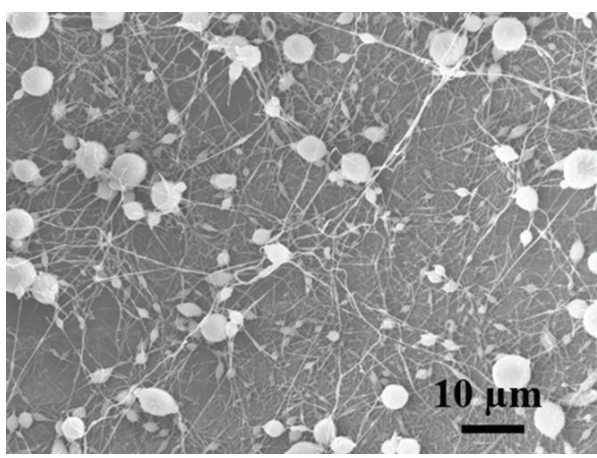

(a)

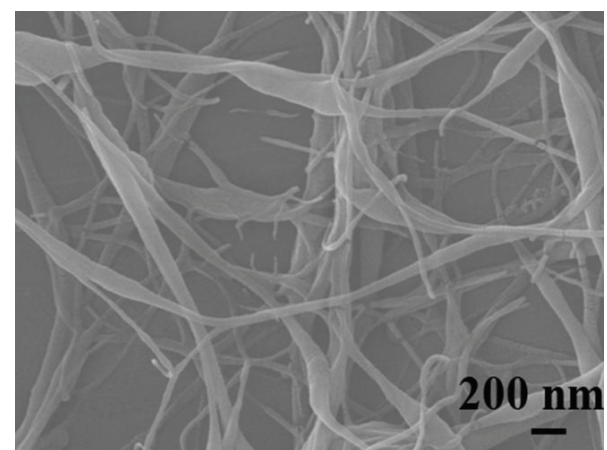

(b)

Figure 2: Beaded PVDF nanofiber electrospun from PVDF solution without surfactants. (a) Electrospun beaded PVDF nanofiber. (b) Close view of beaded nanofiber with magnification of 22,000x.

The diameter and morphology of electrospun nanofiber was observed and measured by SEM. The average diameter was calculated from more than 50 data points in 10 samples.

3.3. Uniform Nanofiber with Surfactants. And then, the effects of surfactant on the diameter and morphology were investigated by adding the surfactants to the polymer solution. The nanofibers electrospun form PVDF solution with surfactants of SDS, Triton X-100, and HTAB were shown in Figures 4-6, respectively. With the increase of surfactant concentration, both the conductivity of polymer solution and the net charge density of electrospinning jet can be increased [18]. The electrical field force applied on the charged jet increased with the increase of net charge density, which also enhanced the instability whipping and spiral motion of charged jets. The instability motion of charged jet was an important role to promote the stretching process of electrospinning jet, decrease the diameter of liquid jet, and prevent the formation of beaded structures in the nanofibers [19]. Therefore, higher surfactant concentration in polymer solution leaded to less beaded structures and more uniform nanofiber. Figures 4-6 showed that the surfactants played a good factor to prevent the formation of beaded structure and increase the uniformity of electrospun nanofiber.

Then, the effects of surfactant concentration on the nanofiber diameter and distribution were studied. The relationships between nanofiber diameter and surfactant concentration in solution were shown in Figure 7. With the help of surfactant, the average nanofiber diameter was less than $65 \mathrm{~nm}$. As surfactant concentration in polymer solution 


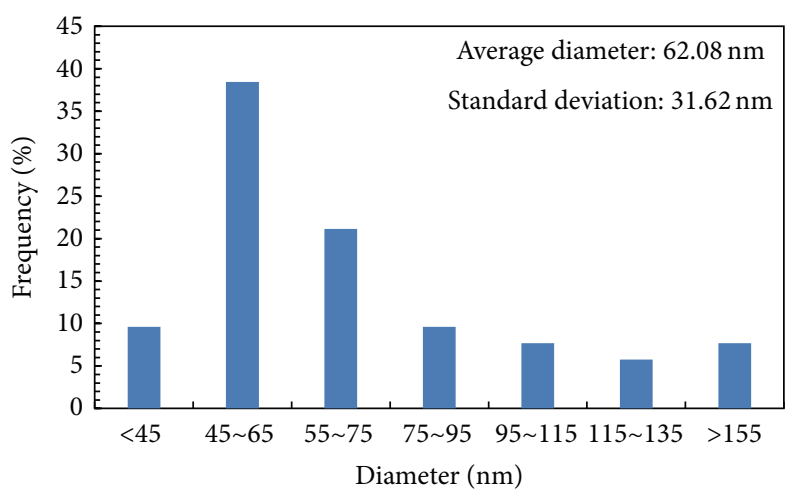

FIgURE 3: The diameter distribution range of nanofiber gained from PVDF solution without surfactant.

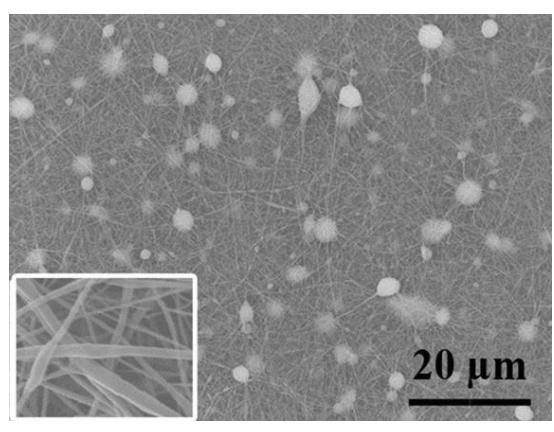

(a)

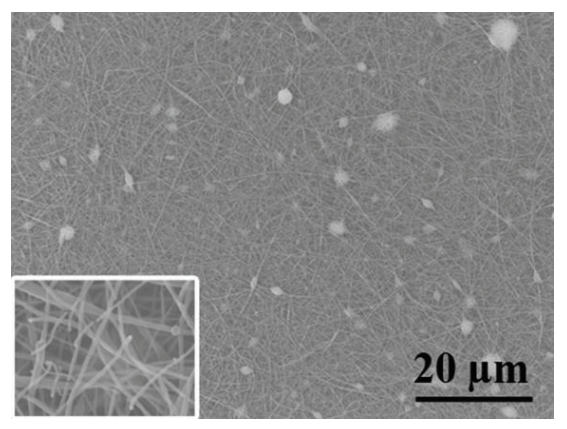

(b)

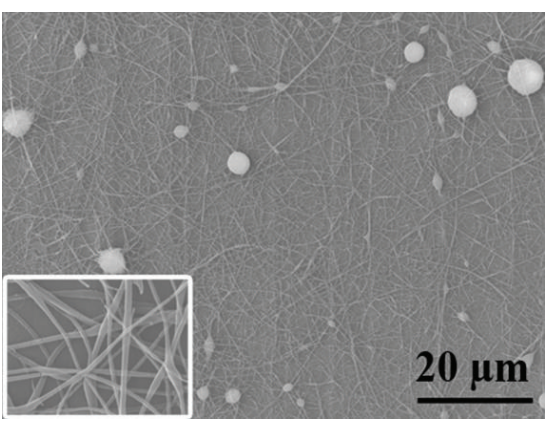

(c)

FIGURE 4: Electrospun nanofiber gained from PVDF solution with anionic surfactants of SDS. The concentration of SDS in the solution is (a) $3.5 \times 10^{-3} \mathrm{~mol} / \mathrm{L}$, (b) $1.75 \times 10^{-2} \mathrm{~mol} / \mathrm{L}$, and (c) $3.5 \times 10^{-2} \mathrm{~mol} / \mathrm{L}$.

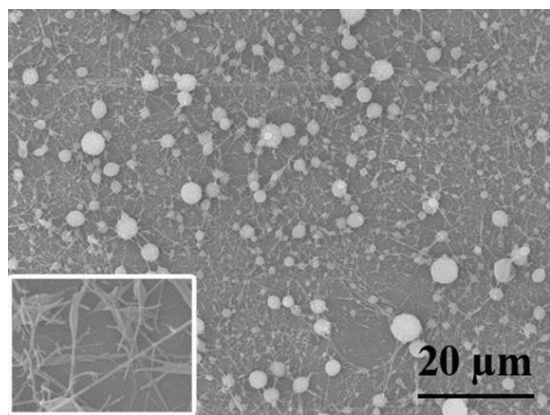

(a)

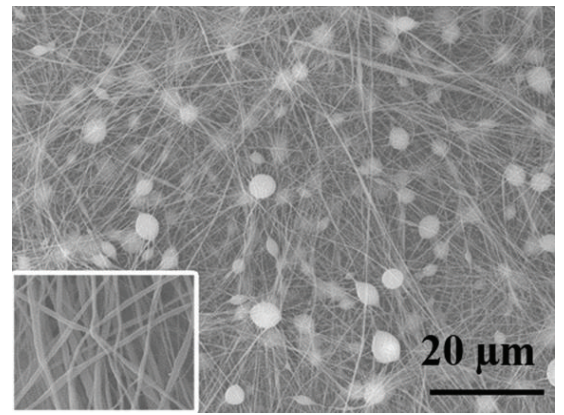

(b)

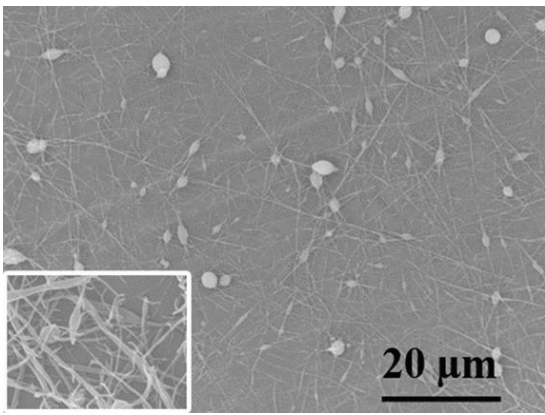

(c)

FIGURE 5: Electrospun nanofiber gained from PVDF solution with nonionic surfactants of Triton X-100. The concentration of Triton X-100 in the solution is (a) $3.5 \times 10^{-3} \mathrm{~mol} / \mathrm{L}$, (b) $1.75 \times 10^{-2} \mathrm{~mol} / \mathrm{L}$, and (c) $3.5 \times 10^{-2} \mathrm{~mol} / \mathrm{L}$.

increased from $3.5 \times 10^{-3} \mathrm{~mol} / \mathrm{L}$ to $3.5 \times 10^{-2} \mathrm{~mol} / \mathrm{L}$, the diameter of PVDF nanofiber with SDS decreased from $51.68 \mathrm{~nm}$ to $39.89 \mathrm{~nm}$, the diameter of PVDF nanofiber with Triton $\mathrm{X}-100$ decreased from $63.91 \mathrm{~nm}$ to $40.62 \mathrm{~nm}$, and the diameter of PVDF nanofiber with HTAB decreased from $60.19 \mathrm{~nm}$ to $41.61 \mathrm{~nm}$. Figures $8-10$ showed the diameter distribution range of nanofiber electrospun from PVDF solution with surfactant. The diameter of nanofiber ranged from $30 \mathrm{~nm}$ to $70 \mathrm{~nm}$. The surfactant concentration in solution was $3.5 \times$ $10^{-3} \mathrm{~mol} / \mathrm{L}$ in Figure 8 . The surfactant concentration in 


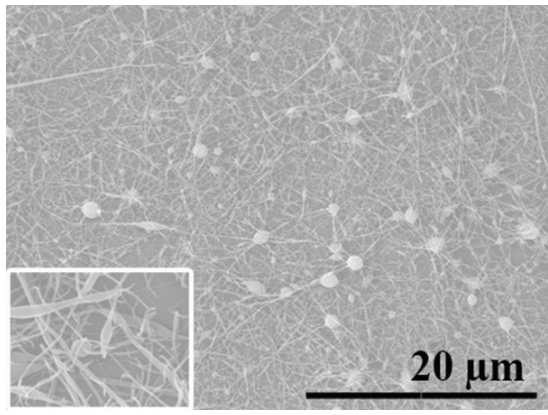

(a)

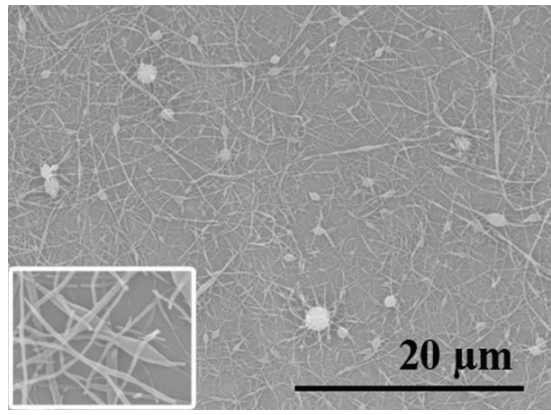

(b)

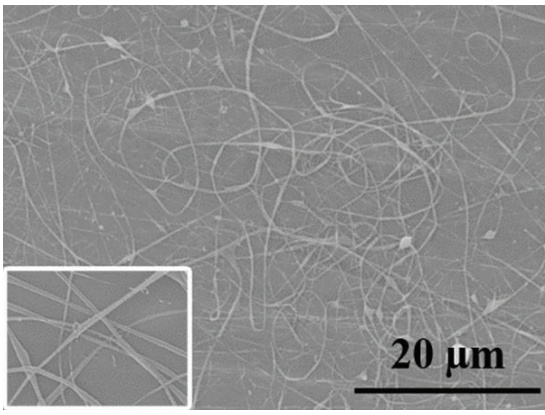

(c)

FIGURE 6: Electrospun nanofiber gained from PVDF solution with cationic surfactants of HTAB. The concentration of HTAB in the solution is (a) $3.5 \times 10^{-3} \mathrm{~mol} / \mathrm{L}$, (b) $1.75 \times 10^{-2} \mathrm{~mol} / \mathrm{L}$, and (c) $3.5 \times 10^{-2} \mathrm{~mol} / \mathrm{L}$.

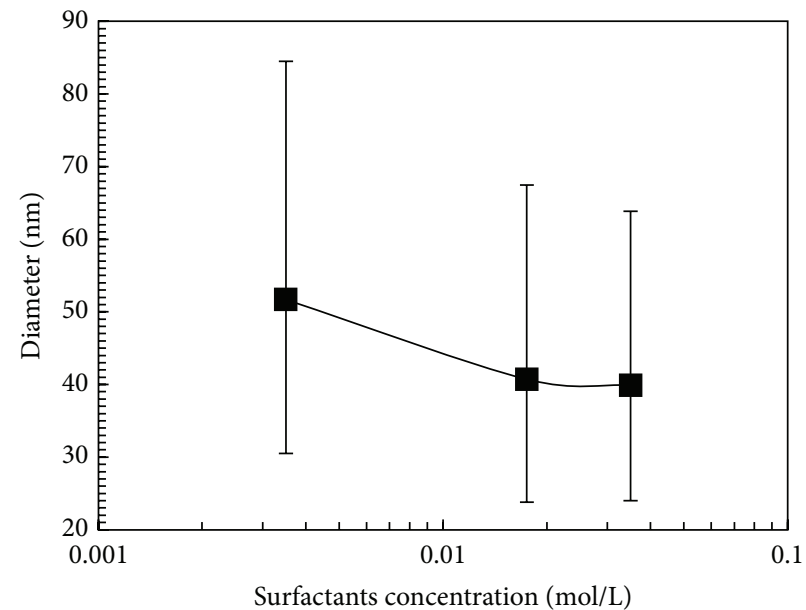

(a)

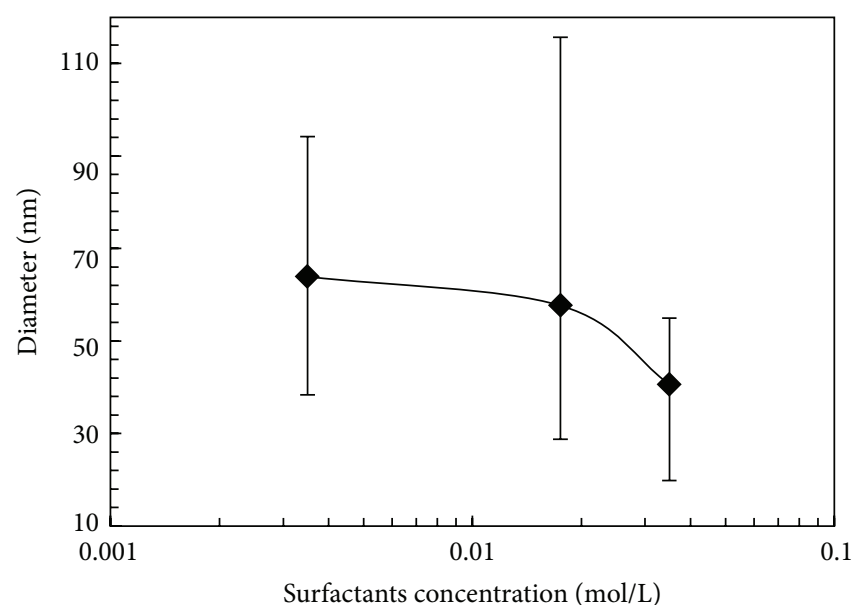

(b)

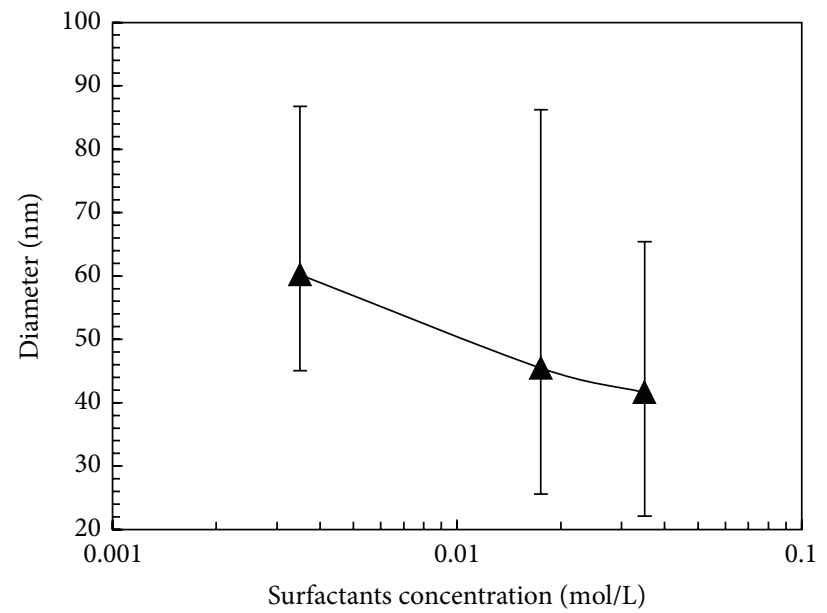

(c)

FIgURE 7: The relationship between nanofiber diameter and surfactant concentration in the solution: (a) SDS, (b) Triton X-100, and (c) HTAB. 


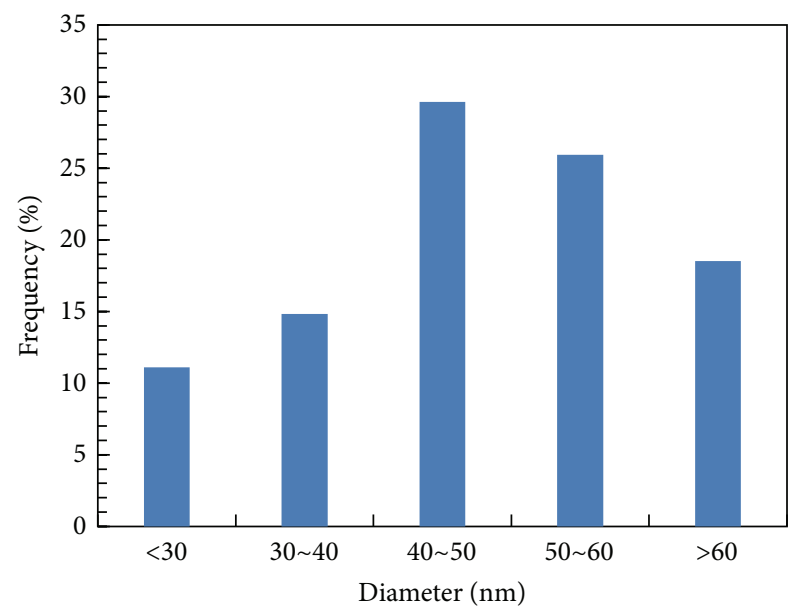

(a)

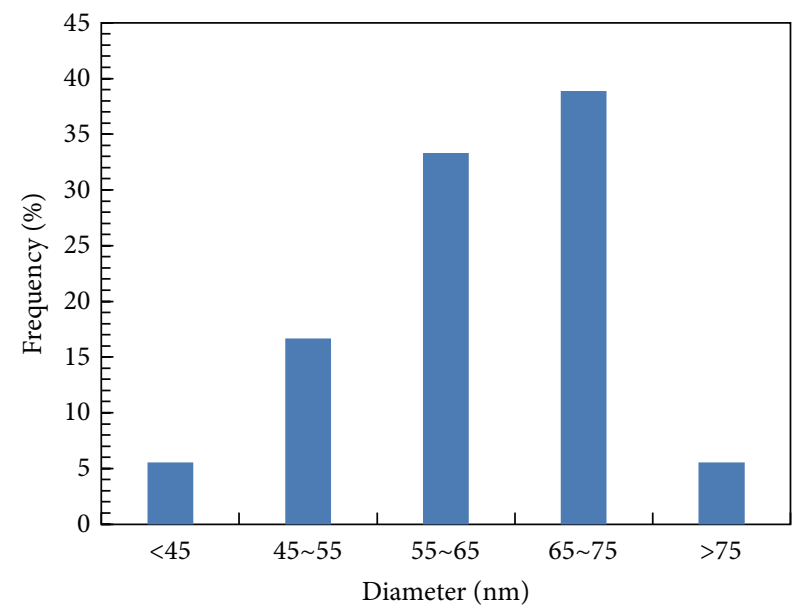

(b)

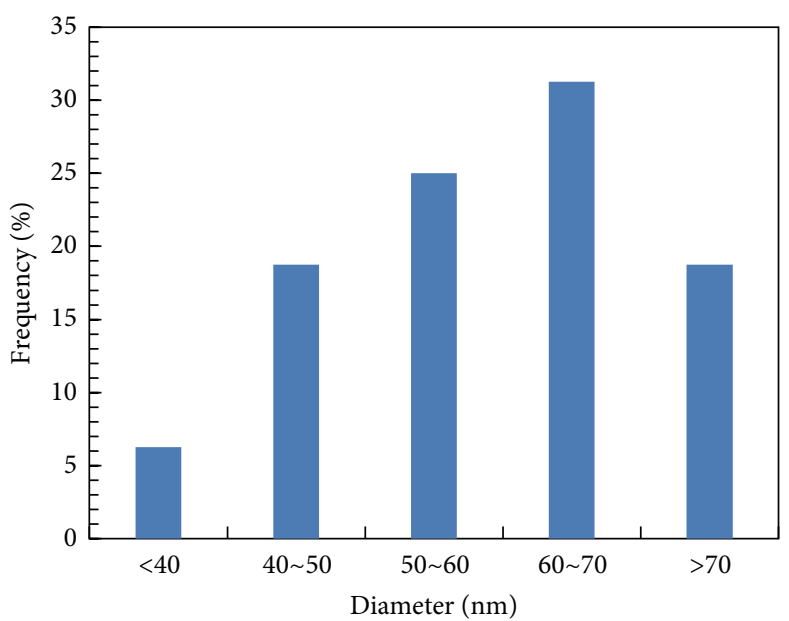

(c)

FIGURE 8: The diameter distribution of nanofiber gained from PVDF solution with surfactants: (a) SDS: average diameter is $51.68 \mathrm{~nm}$ and standard deviation is $13.81 \mathrm{~nm}$; (b) Triton X-100: average diameter is $63.91 \mathrm{~nm}$ and standard deviation is 11.09 nm; (c) HTAB: average diameter is $60.19 \mathrm{~nm}$ and standard deviation is $13.71 \mathrm{~nm}$. The surfactant concentration in solution was $3.5 \times 10^{-3} \mathrm{~mol} / \mathrm{L}$.

solution was $1.75 \times 10^{-2} \mathrm{~mol} / \mathrm{L}$ in Figure 9. The surfactant concentration in solution was $3.5 \times 10^{-2} \mathrm{~mol} / \mathrm{L}$ in Figure 10 . Both of nanofiber diameter and diameter distribution range decreased with the increase of surfactant concentration.

Different surfactants had played different roles in the rheology behaviors of charged jet. The nanofibers gained from PVDF solution with anionic surfactant SDS were shown in Figure 4. There were the least beaded structures in Figure 4(b), when surfactant concentration of SDS was 1.75 $\times 10^{-2} \mathrm{~mol} / \mathrm{L}$. When surfactant concentration of solution was $3.5 \times 10^{-2} \mathrm{~mol} / \mathrm{L}$, nanofibers electrospun from PVDF solution had large beaded structure, as shown in Figure 4(c). Then, the diameter of beaded structure in Figure 4(c) was larger than that in Figures 4(a) and 4(b). The nanofibers gained from PVDF solution with nonionic surfactant of Triton X-100 were shown in Figure 5. Large surfactant concentration led to less beaded structures and smaller diameter. The nanofibers gained from PVDF solution with surfactant of HTAB in Figure 6 had less and smaller beaded structures than that in Figures 4 and 5. The relationship in Figure 7 showed that the nanofiber with the surfactant of SDS had the smallest diameter, attributed to larger charge repulsion force among liquid jets.

In this work, the anode of the high voltage source was connected to the steel nozzle spinneret. When injected from the spinneret, the liquid jet also carried away the positive charge accumulated on the spinneret. The cationic surfactant of HTAB would play a good way to provide excess positive charge to the liquid jet during the injection process. On the other hand, polymer solution with HTAB had the highest 


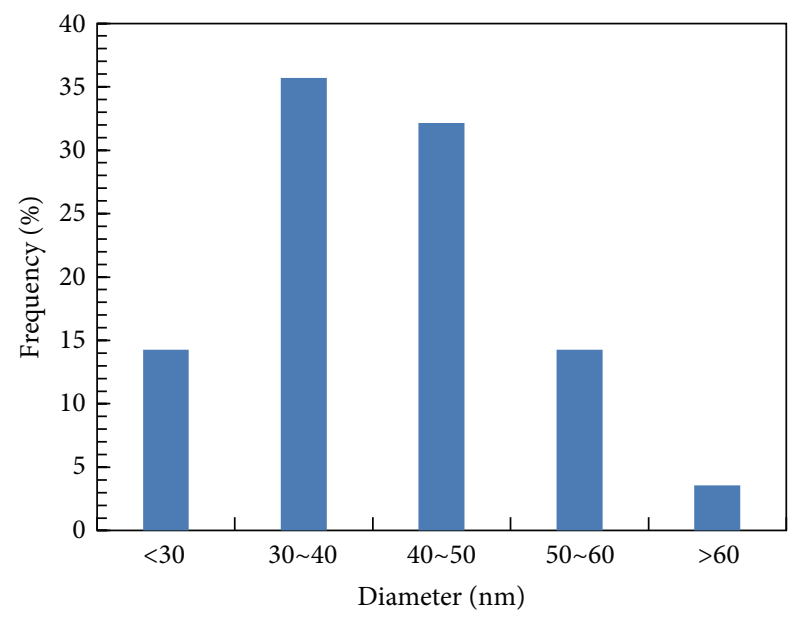

(a)

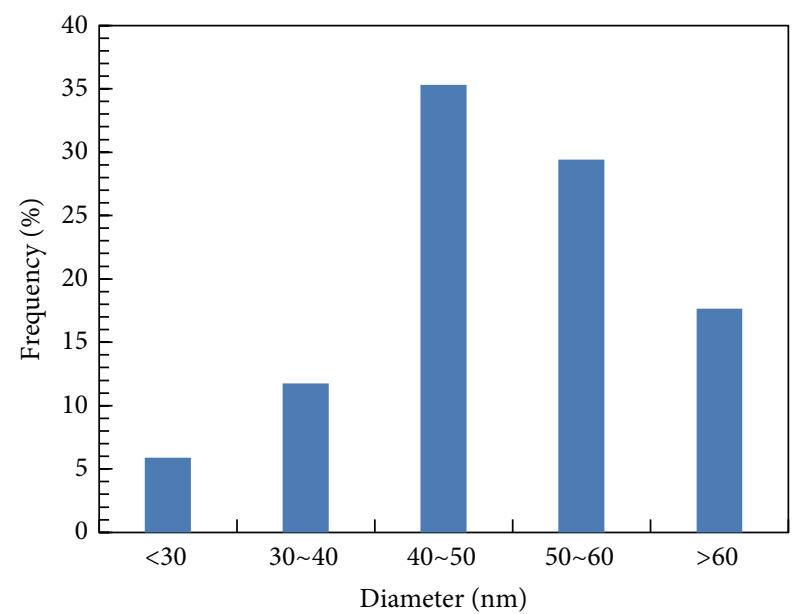

(b)

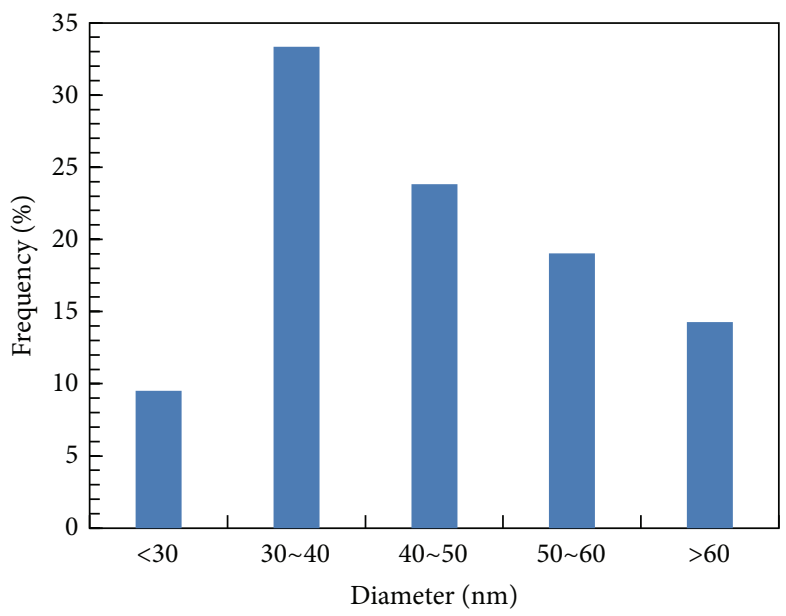

(c)

FIGURE 9: The diameter distribution of nanofiber gained from PVDF solution with surfactants: (a) SDS: average diameter is $40.71 \mathrm{~nm}$ and standard deviation is $10.02 \mathrm{~nm}$; (b) Triton X-100: average diameter is $56.51 \mathrm{~nm}$ and standard deviation is $22.95 \mathrm{~nm}$; (c) HTAB: average diameter is $45.55 \mathrm{~nm}$ and standard deviation is $15.15 \mathrm{~nm}$. The surfactant concentration in solution was $1.75 \times 10^{-2} \mathrm{~mol} / \mathrm{L}$.

conductivity that would increase the free charges in solution. And then, the net charge density can be increased to enhance the instability motion of charged jet. Thus, the nanofiber electrospun from PVDF solution with cationic surfactant of HTAB had the smallest and least beaded structure in nanofiber.

\section{Conclusion}

Different surfactants were introduced to investigate the rheology behaviors of charged jet electrospun from PVDF solution. With the help of surfactants, net charge density in electrospinning jet was increased to enhance the charge repulsion force and the instability motion of charged jet.
Charged jets can be stretched adequately into finer and uniform ones by the larger electrical field force. With the help of surfactants, electrospinning nanofiber with average diameter less than $65 \mathrm{~nm}$ can be fabricated, which was finer than nanofiber electrospun from PVDF solution without surfactant. The nanofiber diameter and diameter distribution range decreased with the increase of surfactant concentration in solution. Attributed to the larger net charge density, the cationic surfactant of HTAB would provide a great way to prevent forming beaded structures.

The effects of surfactant on the transform characteristics of nanofiber diameter and morphology were studied, which would provide a good way to promote the industrial application of polymeric nanofibers. 


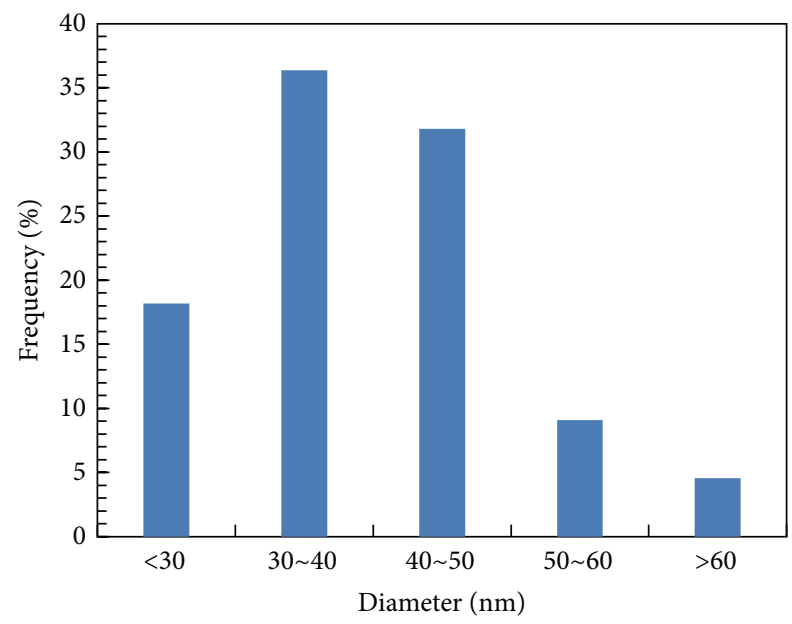

(a)

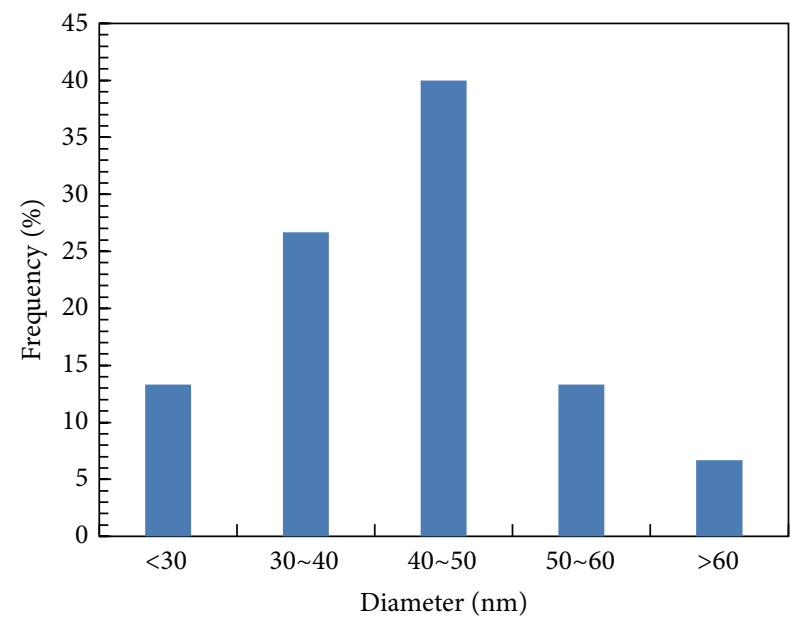

(b)

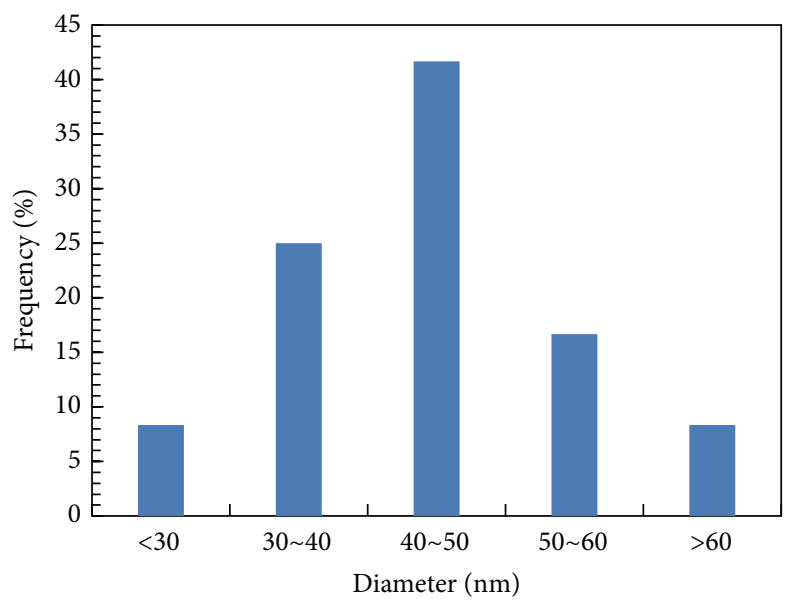

(c)

FIGURE 10: The diameter distribution of nanofiber gained from PVDF solution with surfactants: (a) SDS: average diameter is 39.89 nm and standard deviation is $9.99 \mathrm{~nm}$; (b) Triton X-100: average diameter is $40.62 \mathrm{~nm}$ and standard deviation is $10.24 \mathrm{~nm}$; (c) HTAB: average diameter is $41.61 \mathrm{~nm}$ and standard deviation is $12.88 \mathrm{~nm}$. The surfactant concentration in solution was $3.5 \times 10^{-2} \mathrm{~mol} / \mathrm{L}$.

\section{Conflict of Interests}

The authors declare that there is no conflict of interests regarding the publication of this paper.

\section{Acknowledgments}

This work is supported by theNational Natural Science Foundation of China (nos. 51035002 and 51305371), Major Science and Technology Projects of Fujian Province of China (no. 2012H6022), and Opening fund of Guangdong Provincial Key Laboratory of Micro-Nano Manufacturing Technology and Equipment (no. GDMNML2013-01).

\section{References}

[1] J. C. Bolsée, W. D. Oosterbaan, L. Lutsen, D. Vanderzande, and J. Manca, "The importance of bridging points for charge transport in webs of conjugated polymer nanofibers," Advanced Functional Materials, vol. 23, no. 7, pp. 862-869, 2013.

[2] Y. Xia, P. Yang, Y. Sun et al., "One-dimensional nanostructures: synthesis, characterization, and applications," Advanced Materials, vol. 15, no. 5, pp. 353-389, 2003.

[3] W. Feng, Z. Wu, Y. Li, Y. Feng, and X. Yuan, "The fabrication and electrochemical properties of electrospun nanofibers of a multiwalled carbon nanotube grafted by chitosan," Nanotechnology, vol. 19, no. 10, Article ID 105707, 2008.

[4] L. Persano, A. Camposeo, C. Tekmen, and D. Pisignano, "Industrial upscaling of electrospinning and applications of polymer nanofibers: a review," Macromolecular Materials and Engineering, vol. 298, no. 5, pp. 504-520, 2013.

[5] W. Qian, D. G. Yu, and Y. Li, “Triple-component drug-loaded nanocomposites prepared using a modified coaxial electrospinning," Journal of Nanomaterials, vol. 2013, Article ID 826471, 7 pages, 2013. 
[6] C. Dott, C. Tyagi, L. K. Tomar et al., "A mucoadhesive electrospun nanofibrous matrix for rapid oramucosal drug delivery," Journal of Nanomaterials, vol. 2013, Article ID 924947, 19 pages, 2013.

[7] Y. Zhang, S. Wu, J. Xu et al., "Preparation and performance characterization of electrospun drug loaded poly(vinyl alcohol)/chitosan nanofibrous membrane," Journal of Zhejiang University, vol. 42, no. 6, pp. 644-648, 2013.

[8] W. G. Chen, Q. Zhou, L. N. Xu et al., "Improved methane sensing properties of Co-doped $\mathrm{SnO}_{2}$ electrospun nanofibers," Journal of Nanomaterials, vol. 2013, Article ID 173232, 9 pages, 2013.

[9] C. Wang, D. Chen, and L. Cheng, "Progress in the application of conducting polymer in glucose biosensor," Journal of Biomedical Engineering, vol. 30, no. 5, pp. 1112-1116, 2013.

[10] H. Wang, D. Wu, D. Li et al., "Fabrication of continuous highly ordered mesoporous silica nanofibre with core/sheath structure and its application as catalyst carrier," Nanoscale, vol. 3, no. 9, pp. 3601-3604, 2011.

[11] Y. E. Miao, G. N. Zhu, H. Hou, Y. Y. Xia, and T. Liu, "Electrospun polyimide nanofiber-based nonwoven separators for lithiumion batteries," Journal of Power Sources, vol. 226, pp. 82-86, 2013.

[12] L. Han, C. Lu, P. Chen, W. Qi, and Q. Yu, "The effects of in situ generated titanium dioxide on the mechanical and electrochemical properties of electrospun polyvinylidene fluoride seperator for lithium-ion battery," Acta Polymerica Sinica, no. 11, pp. 1319-1325, 2012.

[13] V. Pillay, C. Dott, and Y. E. Choonara, "A review of the effect of processing variables on the fabrication of electrospun nanofibers for drug delivery applications," Journal of Nanomaterials, vol. 2013, Article ID 789289, 22 pages, 2013.

[14] R. Asmatulu, M. Ceylan, and N. Nuraje, "Study of superhydrophobic electrospun nanocomposite fibers for energy systems," Langmuir, vol. 27, no. 2, pp. 504-507, 2011.

[15] K. H. Lee, H. Y. Kim, H. J. Bang, Y. H. Jung, and S. G. Lee, “The change of bead morphology formed on electrospun polystyrene fibers," Polymer, vol. 44, no. 14, pp. 4029-4034, 2003.

[16] T. Lin, H. Wang, H. Wang, and X. Wang, "The charge effect of cationic surfactants on the elimination of fibre beads in the electrospinning of polystyrene," Nanotechnology, vol. 15, no. 9, pp. 1375-1381, 2004.

[17] L. Jia and X. H. Qin, "The effect of different surfactants on the electrospinning poly(vinyl alcohol) (PVA) nanofibers," Journal of Thermal Analysis and Calorimetry, vol. 112, no. 2, pp. 595-605, 2013.

[18] K. Ziani, C. Henrist, C. Jérôme, A. Aqil, J. I. Maté, and R. Cloots, "Effect of nonionic surfactant and acidity on chitosan nanofibers with different molecular weights," Carbohydrate Polymers, vol. 83, no. 2, pp. 470-476, 2011.

[19] W. Li, G. Zheng, X. Wang et al., "Directly electrospun ultrafine nanofibres with Cu grid spinneret," Journal of Physics D: Applied Physics, vol. 44, no. 13, Article ID 135502, 2011. 

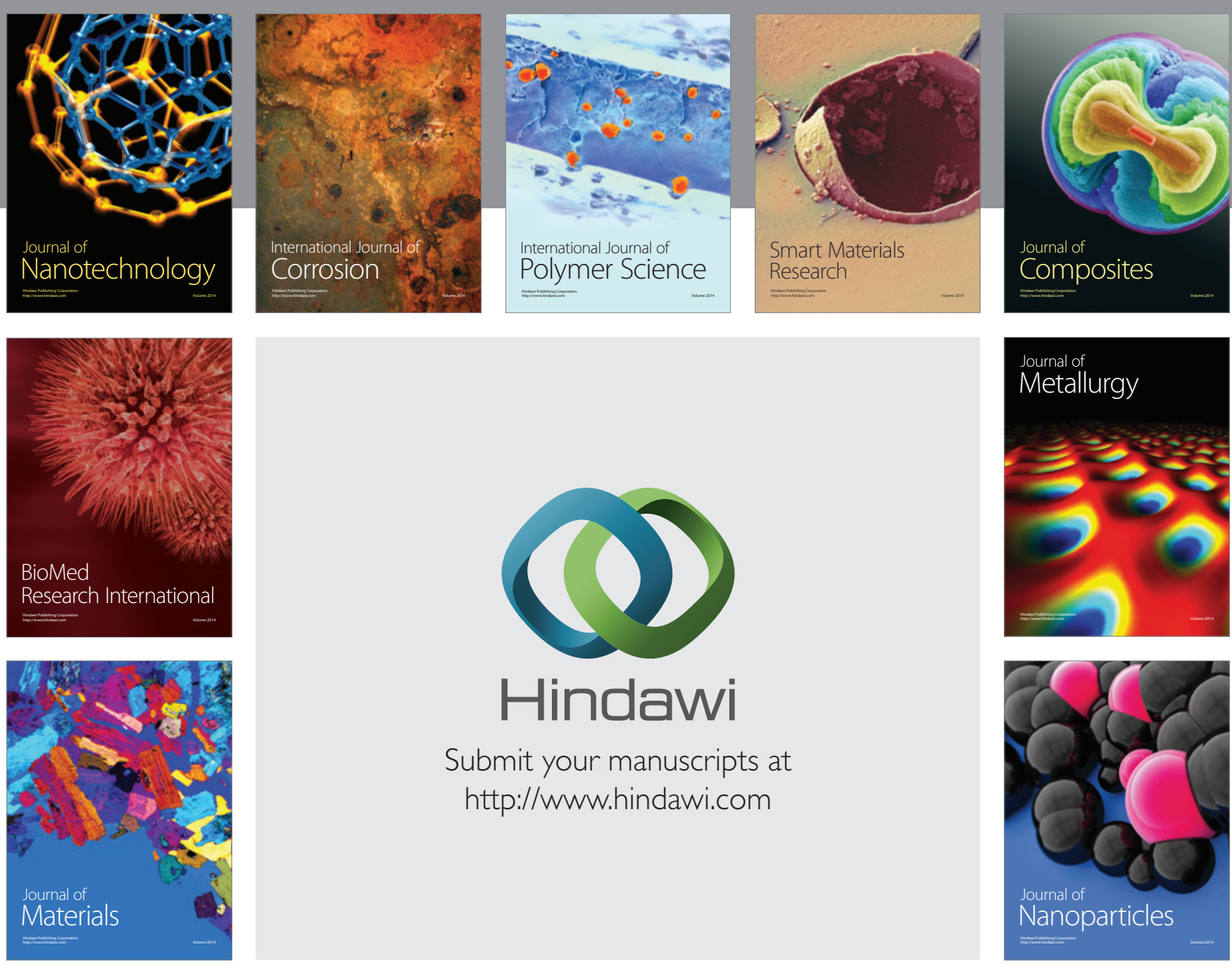

Submit your manuscripts at http://www.hindawi.com
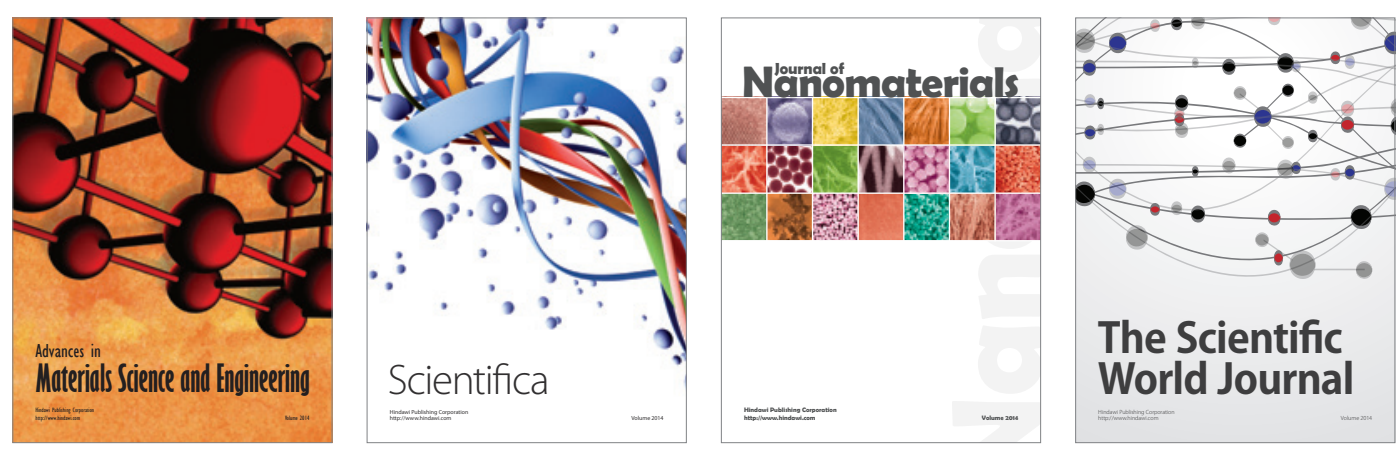

\section{The Scientific World Journal}
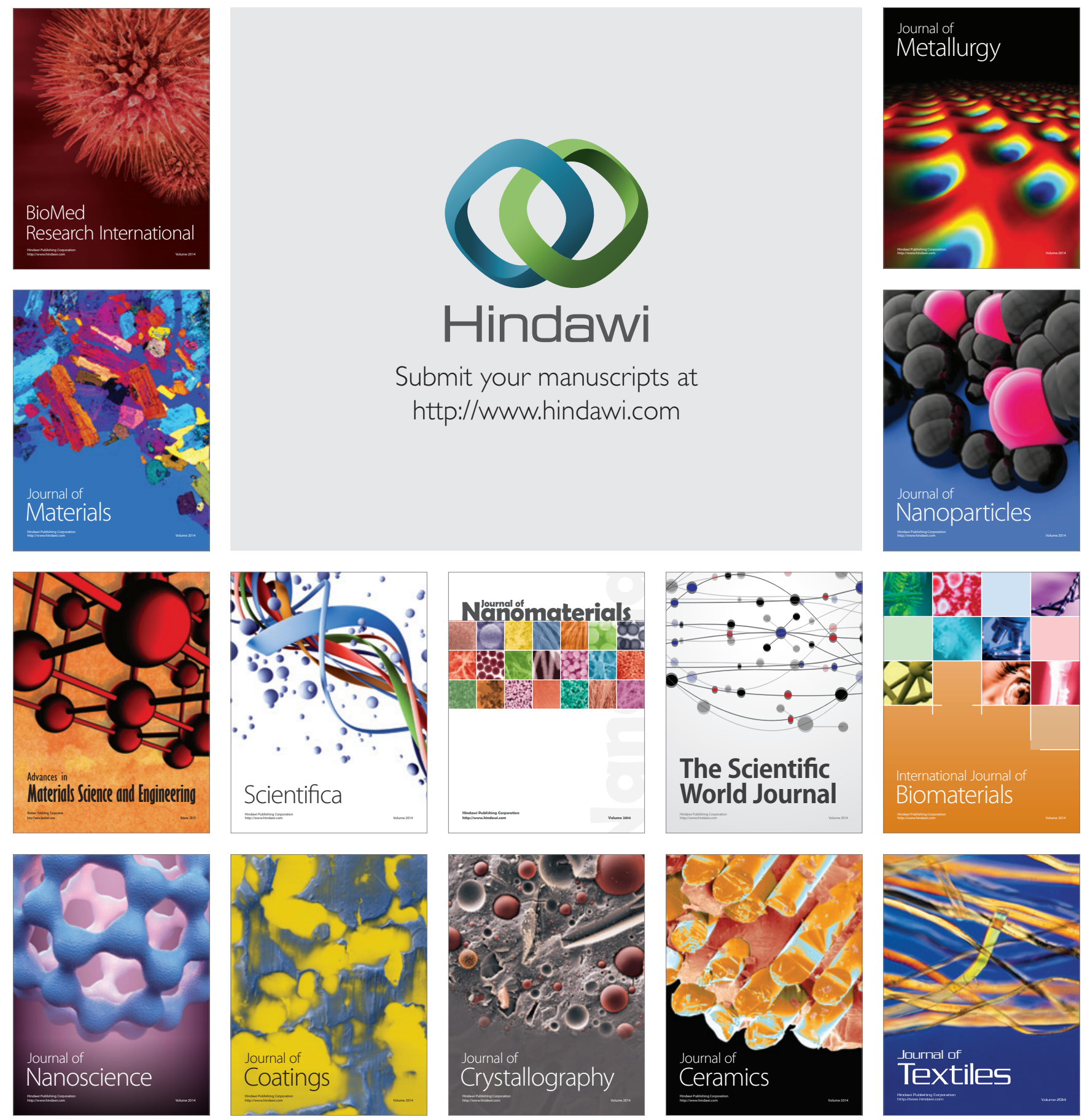